\title{
医用生物相容性接枝共聚物负载阿霉素和香豆素的研究
}

\author{
钱文吴 $a, b$ 苏俭生 $*, a$ 龚 雪 $a$ 叶 茂 ${ }^{b}$ 徐培成 $*, b$ \\ ( ${ }^{a}$ 上海牙组织修复与再生工程技术研究中心 同济大学口腔医学院口腔修复学教研室 上海 200072) \\ $\left({ }^{b}\right.$ 上海市徐汇区牙病防治所 上海 200032)
}

\begin{abstract}
摘要 以一端连有单电子转移自由基聚合( RAFT)链转移剂的聚乙二醇 $(\mathrm{PEG})$ 为大分子链转移剂, 调控 2-(4-差基丁酰氧 基)甲基丙烯酸叔丁酯( $t$ BHBMA)的 RAFT 聚合, 得到的 PEG- $b$-P $t$ BHBMA 嵌段共聚物引发丙交酯的开环聚合, 制得接 枝共聚物 PEG- $b$-(PtBA-g-PLA). 通过聚乳酸末端的羟基与 7-甲氧基香豆素- 3-羧酸(COU)中羧基的酯化反应, 得到了含 有苂光标记分子的接枝共聚物 PEG- $b$-(PtBA-g-PLA-COU). 该聚合物主链选择性水解, 得到了含有苂光标记分子的两 亲性接枝共聚物 PEG- $b$-(PAA- $g$-PLA-COU). 以 PEG- $b$-(PAA- $g$-PLA-COU)为药物载体, 对阿霉素(DOX)进行了负载, 制 得了含有荧光标记分子的聚合物载药胶束. 利用紫外光谱和动态光散射测定了载药胶束的载药量和胶束尺寸.
\end{abstract}

关键词 接枝共聚物; 聚合物载药胶束; 香豆素; 阿霉素

\section{Studies on Loading Doxorubicin and Coumarin by Medical Biocompatible Graft Copolymer}

\author{
Qian, Wenhao $^{a, b} \quad \mathrm{Su}$, Jiansheng*,a $\quad$ Gong, Xue ${ }^{a} \quad \mathrm{Ye}^{*} \mathrm{Mao}^{b} \quad$ Xu, Peicheng ${ }^{*, b}$ \\ ( ${ }^{a}$ Shanghai Engineering Research Center of Tooth Restoration and Regeneration, Department of Prosthodontics, \\ School of Stomatology, Tongji University, Shanghai 200072) \\ ( ${ }^{b}$ Department of Stomatology, Shanghai Xuhui District Dental Center, Shanghai 200032)
}

\begin{abstract}
The end of linear $m$-PEG-OH was functionalized to provide $m$-PEG-CTA macro-RAFT agent. RAFT homopolymerization of $t$ BHBMA was conducted to give PEG- $b$-P $t$ BHBMA diblock copolymer, which initiated ROP of lactide directly. The obtained graft copolymer, PEG- $b$-(PtBA-g-PLA), was then esterified with 7-methoxycoumarin-3- carboxylic acid to afford PEG- $b$-(PtBA- $g$-PLA-COU) containing fluorescent dye molecule. PEG- $b$-(PAA- $g$-PLA-COU) amphiphilic graft copolymer was prepared by selective hydrolysis of PtBA segment. Finally, doxorubicin (DOX) was loaded into polymeric micelles aggregated by PEG- $b$-(PAA- $g$-PLA-COU). The drug loading content (DLC) and size of the obtained polymeric drug micelles containing fluorescent dye molecule was measured by UV-vis and DLS.
\end{abstract}

Keywords graft copolymer; polymeric drug micelles; coumarin; doxorubicin

香豆素(coumarin)又称为 1,2-苯并吡喃酮或香豆精 (结构见图 1), 大量存在于自然界植物中, 是一类重要的 天然产物. 香豆素衍生物具有显著的生物活性和药理活 性, 如抗血栓、抗 HIV 及抗癌等多种药理作用 ${ }^{[1 \sim 4]}$. Weber 等 ${ }^{[5]}$ 通过对 7-羟基香豆素衍生物进行生物活性 测试, 发现该类化合物对人体肿瘤细胞有很好的抑制作 用. 由于香豆素具有特殊的分子结构(同时含有六元杂 环和羰基这两种具有荧光潜能的基团), 并且通过香豆 素环上不同位置的取代基修饰(在其 3-位或 4-位引入吸
电子基团), 如卤原子、氧基、羧基等, 并同时在 7-位引 入供电子基团), 可以得到具有不同范围的吸收和荧光 发射波长、从而显示不同颜色和具有较强荧光的衍生 物 ${ }^{[6]}$. 这些经过不同程度修饰的香豆素类衍生物, 可广 泛用作荧光染料、荧光增白剂、激光染料件、太阳能电 池的有机光敏染料和电致发光材料等 ${ }^{[7 \sim 13]}$. 而且, 香豆 素化合物具有突光量子产率高, Stokes 位移大, 光物理 和光化学性质易于调控以及光稳定性好等优点, 是苂光 探针分子设计中常用的苂光基团. 目前香豆素衍生物常

\footnotetext{
*E-mail: sjs@tongji.edu.cn, xpc1238@126.com
}

Received September 25, 2015; revised October 9, 2015; published online October 13, 2015.

Project supported by the National Natural Science Foundation of China (Nos. 81371949 and 81572114) and the Shanghai Scientific Technological Innovation Project (No. 13411951200).

国家自然科学基金(Nos. 81371949, 81572114)和上海市生物医药科技重点(No. 13411951200)资助项目. 
用于荧光探针技术来检测金属阳离子的存在, 这种检测 方法具有操作简单、灵敏强度高、选择性强和响应时间 短等特点. 同时, 香豆素类苂光探针还可用于细胞的苂 光标记, 并且香豆素类苂光探针对生物酶也有较高的选 择性和灵敏度. 因此, 香豆素类苂光探针技术在医学诊 断、环境化学、分析化学和生物医学科学等领域已被广 泛使用 ${ }^{[14]}$.

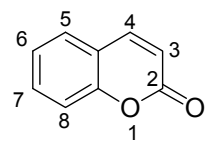

图 1 香豆素结构

Figure 1 Structure of coumarin

两亲性共聚物在水溶液中可自组装形成形貌丰富 的纳米胶束结构, 这些结构在药物输送和靶向载体、纳 米反应器、污水处理、环境净化、电子信息学、催化和 生物模拟等诸多领域 ${ }^{[15 ~ 22] ~}$ 具有潜在的应用前景. 其中, 聚合物胶束作为药物载体具有其独特的优势, 核壳结构 的高分子组装纳米颗粒在水相中具有极好稳定性、临界 聚集浓度低、抗稀释性强、疏水微区可以有效地对肿瘤 治疗药物增溶并实现复合包封. 同时, 其表面的亲水壳 层可以有效保护药物不被生物活性物质所降解, 提高载 体在体内的循环寿命 ${ }^{[23]}$. 而且利用高分子链结构的多 样性反应引入活性基团, 可以更容易地在高分子纳米颗 粒表面连接靶向分子; 或通过引入功能基团使载体对环 境变化或外界刺激具有响应特性, 实现其按需定位给药 的目的, 为实现多重功能载体的制备提供可能.

为了制备生物相容性的药物载体, 聚合物胶束主要 由具有生物相容性和可降解的链段组成. 聚合物纳米药 物载体的原料分为生物聚合物和合成聚合物两类, 目前 已开发的包括明胶、聚酯纤维、聚酸酐、聚酯等数十种. 其中, 真正在生物医学上可靠、经过美国食品药品管理 局(FDA)批准的合成聚合物有聚乳酸(PLA)、聚乙醇酸 $(P G A) 、$ 聚己内酯(PCL)、聚乙二醇(PEG)、聚对二氧环 己酮(PPDO)、聚三亚甲基碳酸酯(PTMC)以及它们之间 的共聚物如聚乳酸聚羟基乙酸(PLGA)等, 均可通过不 同方法制备纳米粒子. 其中, 聚乙二醇由于水溶性好、

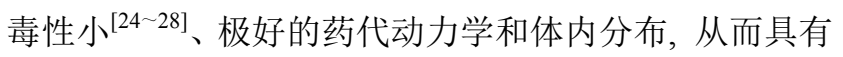
很好的生物相容性. 因此, 为了降低静脉给药后粒子被 血浆清除的速率, 人们常将纳米粒子聚乙二醇化 (PEGylation), 如 “隐形” 脂质体, 能够逃避网状内皮系 统(RES)的迅速摄取, 延长血浆半衰期. 聚乳酸已被广 泛用于纳米粒子和微球以传递多种药物, 如细胞毒剂和 抗疮药 ${ }^{[29]}$ 等, 其制剂在皮下和其他注射部位均具有优 异的生物相容性 ${ }^{[30]}$.
与线性共聚物相比，接枝共聚物的侧链是通过化学 键连接到线性主链上的, 高分子侧链被局限于一定区域 内，因而，接枝共聚物具有高度密集的分子结构，分子 结构的差异使得接枝共聚物表现出很多不同的性 质 ${ }^{[31 ~ 33]}$, 并且接枝共聚物结构复杂性的增大使得影响 多分子胶束形貌和尺寸的因素增多，从而提供了更多的 调控手段。通常采用三种合成策略来合成接枝共聚 物 ${ }^{[34]}$ : 直接接枝法 (grafting-through)、偶合接枝法 (grafting-onto)和引发接枝法(grafting-from).

直接接枝法: 通过大分子单体均聚或与小分子单体 共聚的方法来制备接枝聚合物。该策略制备的接枝共聚 物接枝效率较高, 共聚物组成可以通过调节大分子单体 和小分子单体的投料比进行控制, 有利于进行高分子的 分子设计 ${ }^{[35 ~ 37]}$. 但是, 该方法存在大分子单体很难完全 转化的问题, 通常需要通过后续的分离提纯操作将残留 的大分子单体从产物中分离出去, 过程耗时繁琐.

偶合接枝法首先通过活性聚合及必要的官能团转 化, 分别得到链上官能化 $(\mathrm{X})$ 的主链和末端官能化 $(\mathrm{Y})$ 的 侧链, 然后将侧链通过偶联反应 $(\mathrm{X}$ 和 $\mathrm{Y}$ 间的反应)接枝 到主链上, 形成接枝共聚物. 该方法的优点在于由于主 链和侧链可以分别通过活性聚合合成, 因此可以很好地 控制其结构和组成 ${ }^{[38,39]}$. 利用这种方法接入长侧链的接 入效率通常不高, 并且这种方法很难控制接枝密度, 近 些年来, 随着以点击化学为代表的一系列简便、高效的 偶合技术的出现，使得偶合接枝法的不足之处得到了极 大的改善 ${ }^{[40,41]}$.

引发接枝法首先要合成具有明确结构的主链, 并且 主链上含有可以进一步修饰的官能团, 然后通过不同的 修饰方法, 在主链上引入不同的引发官能团, 接着引发 相应单体得到合适的侧链，从而制得接枝共聚物. 随着 活性可控聚合特别是活性可控自由基聚合的发展，如可 控开环聚合 (controlled ring-opening polymerization, $\mathrm{CROP}^{[42]}$ )、氮氧调控聚合(nitroxide-mediated polymerization, $\mathrm{NMP}^{[43]}$ )、原子转移自由基聚合(atom transfer radical polymerization, ATRP ${ }^{[44,45]}$ 、单电子转移自由基聚 合(single-electron transfer living radical polymerization, SET-LRP ${ }^{[46,47]}$ ) 和可逆加成裂解链转移聚合 [reversible addition fragmentation chain transfer (RAFT) polymeriza$\left.\operatorname{tion}^{[48 \sim 50]}\right]$, 引发接枝法逐渐被认为是制备接枝共聚物 最为有效的策略. 通过活性可控自由基聚合合成侧链, 一方面，低浓度的活性侧链可有效抑制活性侧链的终止 反应以及链间的双基终止反应; 另一方面，“缓慢”增 长的侧链可以避免聚合物链间的位阻效应, 而这在向主 干接枝法和直接接枝法中是不可避免的. 此外, 由于反 应后聚合体系中不存在未反应的大分子引发剂及大分 
子副产物, 所以无须费时和繁琐的纯化过程; 而对于一 些具有复杂结构的接枝共聚物而言, 所用到的合成策略 可能不仅仅局限于其中的一种, 可能会将这几种策略组 合使用.

本文中, 我们结合 RAFT 和 CROP 活性聚合技术, 制备得到含 PEG 和 PLA 生物相容性链段的接枝共聚物, 并引入苂光标记分子 7-甲氧基香豆素-3-羧酸(COU), 接 着选择性水解得到含苂光标记分子 $\mathrm{COU}$ 的两亲性接枝 共聚物, 为药物载体的种类和体内分布研究提供了新的 模型聚合物(Scheme 1). 首先, 通过线性聚乙二醇单甲 醚的末端官能化, 得到 PEG 化的大分子链转移试剂 $m$ PEG-CTA (1), 然后以其为 RAFT 聚合的链转移试剂, 调控 2-(4-差基丁酰氧基)甲基丙烯酸叔丁酯( $t$ BHBMA)
单体的 RAFT 聚合, 得到了两嵌段共聚物 PEG- $b$ PtBHBMA (2)大分子引发剂, 以 PEG- $b-\mathrm{P} t \mathrm{BHBMA}$ (2)为 大分子引发剂, 直接引发丙交酯(LA)开环聚合后得到了 接枝共聚物 PEG- $b$-(PtBA- $g$-PLA) (3). 接着, 利用 PEG$b$-(PtBA- $g-\mathrm{PLA})$ 侧链 PLA 末端的羟基与 COU 中羧基的 酯化反应，得到含有苂光标记分子 $\mathrm{COU}$ 的接枝共聚物 PEG- $b$-(PtBA- $g$-PLA-COU) (4). 随后，以三氟乙 酸 (TFA)为催化剂, 选择性水解掉主链中的叔丁基, 得到 主链为 PEG 和聚丙烯酸(PAA)的两亲性接枝共聚物 PEG- $b$-(PAA- $g$-PLA-COU) (5). 最后, 以 PEG- $b$-(PAA$g$-PLA-COU) (5)为载体, 研究了该聚合物对 DOX 的负 载情况.

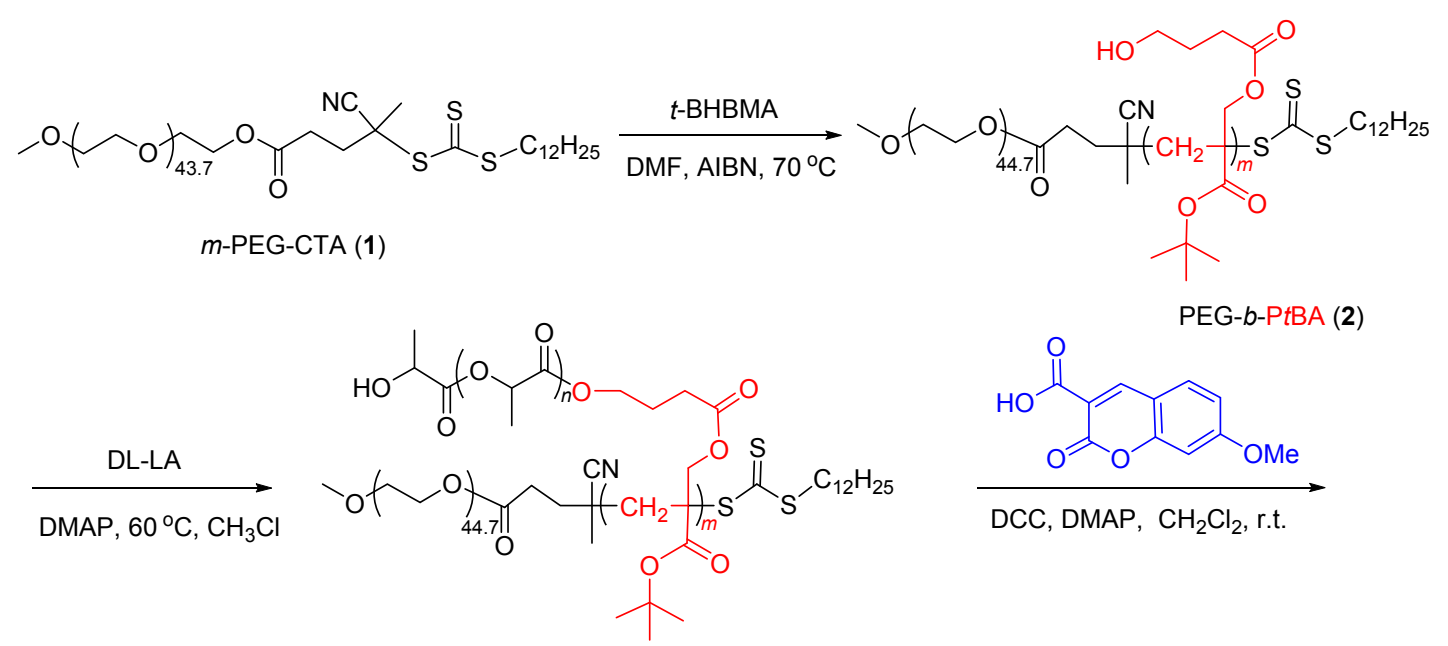

PEG-b-(PtBA-g-PLA) (3)

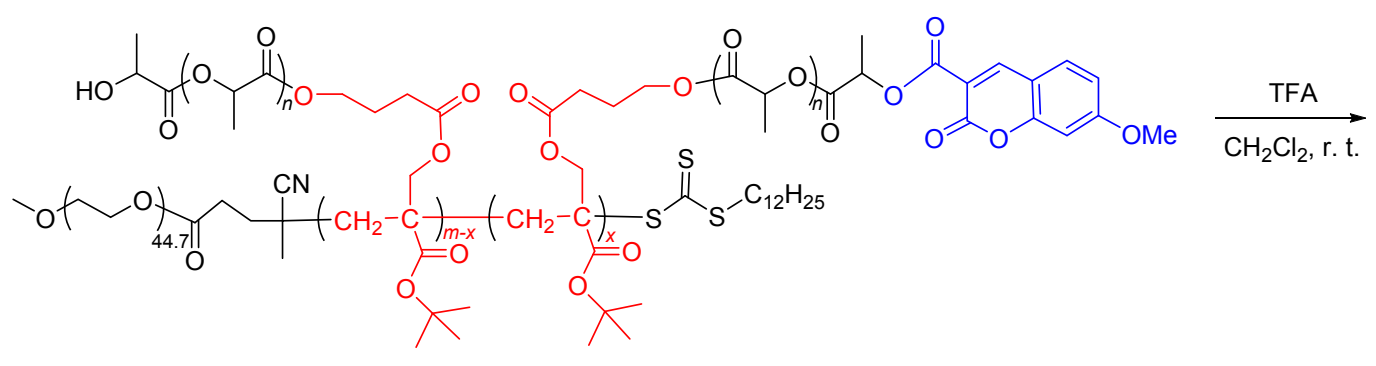

PEG-b-(PtBA-g-PLA-COU) (4)

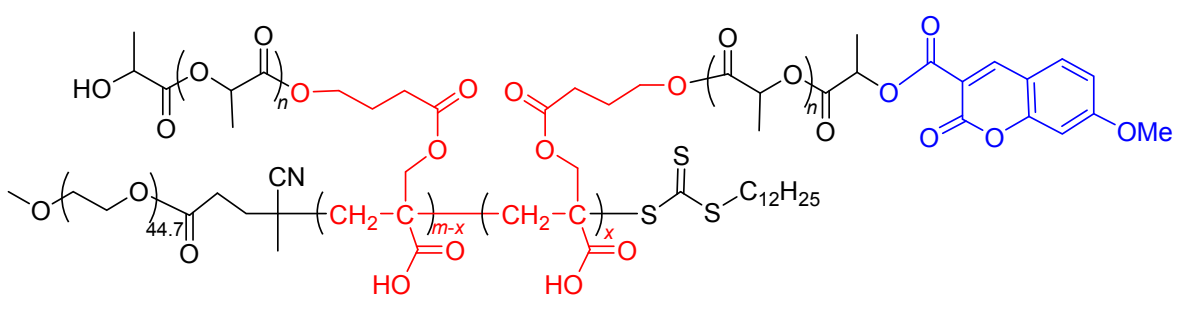

PEG- $b$-(PAA-g-PLA-COU) (5)

图式 1 两亲性接枝共聚物 PEG- $b$-(PAA- $g$-PLA-COU) (5)的合成

Scheme 1 Synthesis of amphiphilic graft copolymer PEG- $b$-(PAA-g-PLA-COU) (5) 


\section{1 结果与讨论}

\subsection{PEG- $b$-(PtBA- $g$-PLA)接枝共聚物的合成}

首先根据文献 $[51,52]$, 利用三硫代碳酸酯类 RAFT 试剂 4-㲵基-4-(十二烷硫基硫代羰基)硫基戊酸末端的羧 基与聚乙二醇单甲醚 $\left(M_{\mathrm{n}}=2000 \mathrm{~g} / \mathrm{mol}\right)$ 的末端羟基的酯 化反应, 得到了末端连有链转移试剂的聚环氧乙烷大分 子链转移试剂 (m-PEG-CTA, 1). 随后, 以 AIBN 为引发 剂, 利用 $m$-PEG-CTA 调控单体 2-溴异丁酰氧基甲基丙 烯酸叔丁酯 $($ BHBMA) 的 RAFT 聚合, 得到了两嵌段大 分子引发剂 PEG- $b$-PtBHBMA (2). 其 GPC 曲线为对称 窄分布的单峰(图 4), 分子量也明显大于相应的大分子 链转移试剂的分子量. 通过 ${ }^{1} \mathrm{H}$ NMR 确认了 PEG- $b$-P $t$ BHBMA (2)的结构. 图 2 所示的 ${ }^{1} \mathrm{H}$ NMR 谱图 中同时存在 $P E G$ 和 $\mathrm{P} t \mathrm{BHBMA}$ 链段的信号峰, 其中 $\mathrm{PEG}$ 链段的亚甲基峰在 $\delta 3.62$ 处，相对高场处的“h,k,n”峰均 属于 $\mathrm{P} t \mathrm{BHBMA}$ 链段; 而 $\delta 3.35$ 处是 RAFT 试剂中与 $\mathrm{S}$ 原子相连的亚甲基峰. 以上结果表明, 我们通过大分子 链转移剂 $m$-PEG- CTA, 成功地调控了单体 $t$ BHBMA 的 RAFT 聚合, 合成了含有 ROP 引发基团 $\mathrm{OH}$ 的大分子引 发剂 PEG- $b$ - P $t$ BHBMA (2).

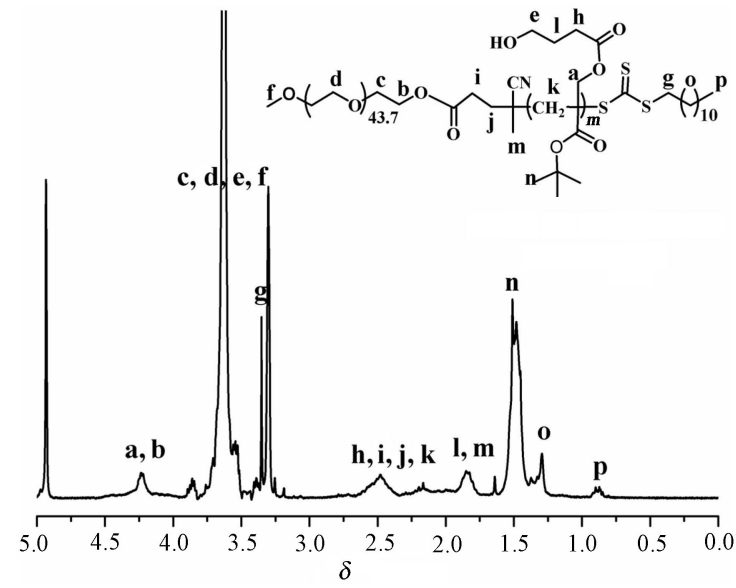

图 2 PEG- $b$-P $t$ BHBMA (2)两嵌段共聚物在忥代甲醇中的核 磁氢谱

Figure $2{ }^{1} \mathrm{H}$ NMR spectrum of PEG- $b$-P $t$ BHBMA (2) diblock copolymer in $\mathrm{CD}_{3} \mathrm{OD}$

接着, 以 PEG- $b-\mathrm{P} t \mathrm{BHBMA}(2)$ 为大分子引发剂, 4二甲氨基吡定(DMAP)为催化剂, $60{ }^{\circ} \mathrm{C}$ 下在 $\mathrm{CHCl}_{3}$ 中反 应 $24 \mathrm{~h}$, 制得了接枝共聚物 PEG- $b$-(PtBA- $g$-PLA) (3). 与 PEG- $b$-PtBHBMA (2) 的氢谱相比, 在接枝共聚物 PEG- $b$-(PtBA- $g$-PLA) (3) 的 ${ }^{1} \mathrm{H}$ NMR 谱图(图 3)中, 明显 多出了新的“a”( $\delta$ 5.13) 和“l” ( $\delta$ 1.56)峰, 分别属于 PLA 的次甲基和甲基, 并且相对于丙交酯单体中的次甲基和 甲基峰，分别向低场和高场发生了移动. 同时，可以看
到主链 $\mathrm{PEG}$ 嵌段和聚丙烯酸叔丁酯( $\mathrm{P} t \mathrm{BA})$ 嵌段的特征 峰，出现在 $\delta 3.62$ 处的亚甲基峰和 $\delta 1.42$ 处的叔丁基特 征峰. 图 4 中接枝共聚物 PEG- $b$-(PtBA- $g$-PLA) (3)的 GPC 流出曲线为窄分布的单峰，分子量明显大于相应的 引发剂. 由此说明，我们成功地合成了结构规整的刷状 接枝共聚物 PEG- $b$-(PtBA- $g$-PLA) (3).

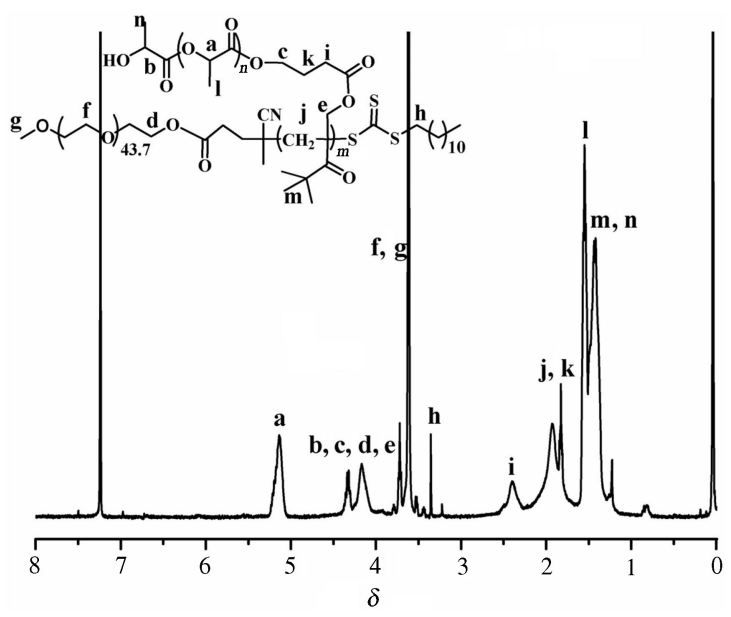

图 3 PEG- $b-(\mathrm{P} t \mathrm{BA}-g$-PLA) (3)接枝共聚物在気代氯仿中的核 磁氢谱

Figure $3 \quad{ }^{1} \mathrm{H}$ NMR spectrum of PEG- $b$-(PtBA- $g$-PLA) (3) graft copolymer in $\mathrm{CDCl}_{3}$

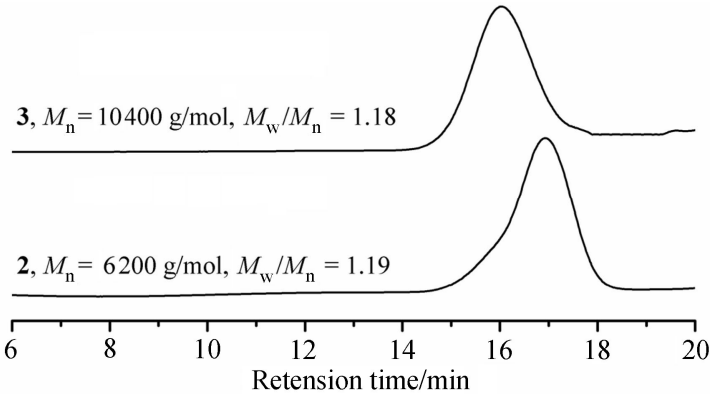

图 4 PEG- $b$-P $t$ BHBMA (2) 和 PEG- $b$-(PtBA- $g$-PLA) (3) 的 GPC 曲线(THF 相)

Figure 4 GPC curves of PEG- $b$-P $t$ BA (2) and PEG- $b$-(PtBA- $g-$ PLA) (3) in THF

\section{2 荧光分子标记的两亲性接枝共聚物 PEG- $b-(P A A-$ $g$-PLA-COU)的合成}

小分子药物存在的诸多缺点, 尤其是抗癌药物存在 溶解性差，毒副作用大，药物在体内稳定性差，药代动 力学不理想和体内分布选择性差等问题 ${ }^{[53]}$. 聚合物载体 通常包括亲水和疏水两部分，疏水部分的疏水环境可增 溶药物, 提高药物的溶解性; 亲水部分包封在疏水部分 的外围，使药物免受水解和酶解，延长药物在血液中循 环时间，提高药物的生物利用度. 通过改变聚合物载体 的组成或在药物载体上接入靶向基团，可通过增强渗透 
滞留效应(enhanced permeability and retention effect, $E P R)^{[54]}$ 和配体-受体间的特异 ${ }^{[5]}$ 结合实现肿瘤组织靶向 性. 所以对于聚合物载体, 我们要追踪这些聚合物胶束 载体在体内的分布情况, 以分析该聚合物载体靶向传输 的有效性，以保证药物对肿瘤组织的疗效.

我们将 7-甲氧基香豆素-3-羧酸(COU)引入到聚合 物 PEG- $b$-(PtBA- $g$-PLA) (3)中. 通过共聚物 3 中 PLA 侧 链末端的羟基与 $\mathrm{COU}$ 中羧基的酯化反应, 得到了含有 $\mathrm{COU}$ 荧光标记分子的接枝共聚物 PEG- $b$-(PtBA- $g$-PLACOU) (4). 与 PEG- $b-(\mathrm{P} t \mathrm{BA}-g-\mathrm{PLA})$ (3)的氢谱(图 3)相比, PEG- $b$-(PtBA-g-PLA-COU) (4) 的 ${ }^{1} \mathrm{H}$ NMR 谱图(图 5)中 出现了苂光分子的所有特征峰, 包括在 $\delta 8.60$ (a), $\delta 7.53$ (b), $\delta 6.91$ (c) 和 $\delta 6.82$ (d) 处出现的苯并吡喃酮上的特征 峰, 在 $\delta 3.91$ 处出现的甲氧基特征峰, 并且在 $\delta 5.35$ (e) 处出现了一个新峰, 这是与荧光分子相连的 PLA 末端 的次甲基峰, 相对于原有的次甲基峰, 向低场发生了少 许位移. 此外, 引入苂光分子前后的共聚物的 GPC 流出 曲线没有明显变化. 以上结果表明 7-甲氧基香豆素-3-羧 酸被成功引入到接枝共聚物 PEG- $b$-(PtBA- $g$-PLA-COU) (4).

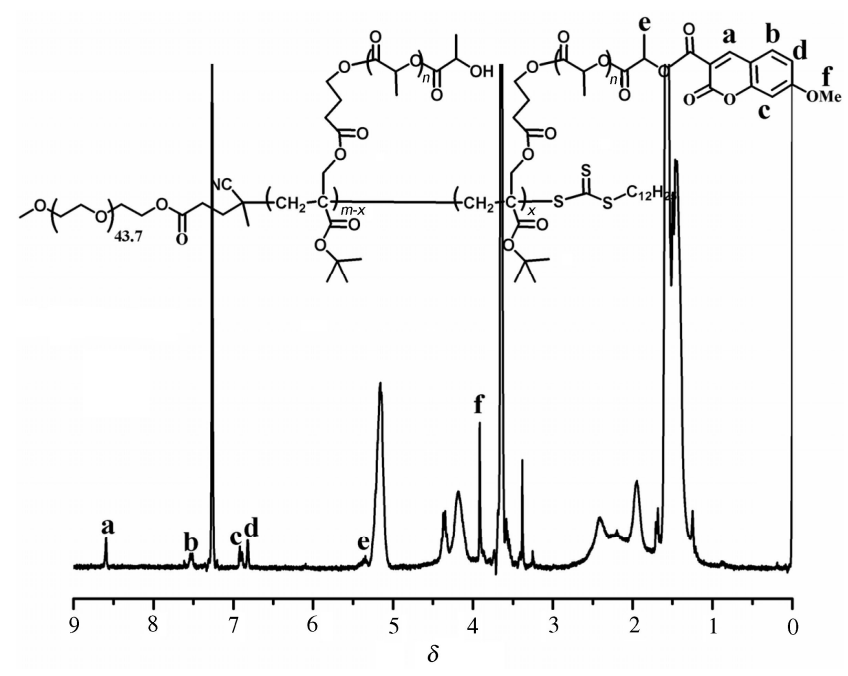

图 5 PEG- $b-(\mathrm{P} t \mathrm{BA}-g-\mathrm{PLA}-\mathrm{COU})$ (4)在気代氯仿中的氢谱

Figure $5{ }^{1} \mathrm{H}$ NMR spectrum of PEG- $b$-(PtBA-g-PLA-COU) (4) in $\mathrm{CDCl}_{3}$

最后, 在三氟乙酸的存在下, 对 PEG- $b-(\mathrm{P} t \mathrm{BA}-g-$ PLA-COU) (4)主链中的叔丁基进行选择性水解, 得到了 含有 PEG、PAA 亲水链段和 PLA 疏水链段的两亲性接 枝共聚物 PEG- $b$-(PAA- $g$-PLA-COU) (5). 我们测得该接 枝共聚物的荧光光谱(图 6), 其最大发射波长为 $405 \mathrm{~nm}$ (激发波长为 $350 \mathrm{~nm}$ ), 进一步说明荧光分子 7-甲氧基香 豆素-3-羧酸的成功引入.

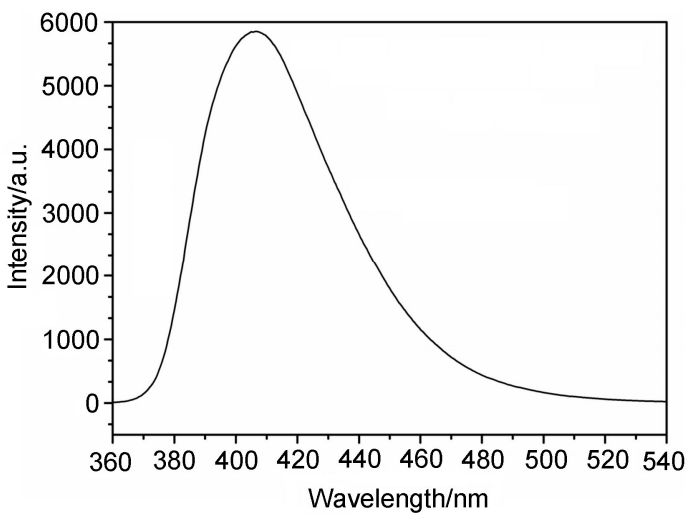

图 6 PEG- $b$-(PAA- $g$-PLA-COU) (5)的 DMF 溶液的荧光光谱(1 $\mathrm{mg} / \mathrm{mL}$ )

Figure 6 Fluorescence spectrum of PEG- $b$-(PAA- $g$-PLA-COU) (5) in DMF with a concentration of $1 \mathrm{mg} / \mathrm{mL}$

\section{3 接枝共聚物 PEG- $b$-(PAA-g-PLA-COU) (5)对 DOX 的负载}

我们采用接枝共聚物 PEG- $b$-(PAA- $g$-PLA-COU) (5) 与 $\mathrm{DOX}$ 溶液共组装的方法制备了负载 $\mathrm{DOX}$ 的聚合物胶 束: 先将 $10 \mathrm{mg}$ PEG- $b$-(PAA-g-PLA-COU) (5)共聚物充 分溶解于 $\mathrm{THF}$ 中, 再向其中加入 $100 \mu \mathrm{L}$ 浓度为 10 $\mathrm{mg} / \mathrm{mL}$ 的 DOX/DMF 溶液, 充分摚拌均匀后, 摚拌下将 混合溶液滴加至 $10 \mathrm{~mL}$ 双蒸水中, 室温搅拌 $12 \mathrm{~h}$, 使 THF 部分挥发后转入透析袋中透析 $24 \mathrm{~h}$, 除去未载入的 药物, 即得到载药胶束水溶液.

为了测定载药量, 我们测定了 DOX 在 DMF 中的标 准曲线. 首先精确配制 $1 \sim 100 \mu \mathrm{g} / \mathrm{mL}$ 的 DOX/DMF 溶 液, 再测定不同浓度的 DOX/DMF 溶液在 $565 \mathrm{~nm}$ 处苂 光发射光谱强度(激发波长为 $535 \mathrm{~nm}$ ), 以测得的数据绘 制强度一浓度曲线(图 7). 该浓度范围内, DOX 在 $565 \mathrm{~nm}$ 处的荧光强度与浓度成线性关系. 按回归法处理得到标 准曲线方程 $\left(I=-0.574+2.327 C_{\mathrm{DOX}}\right)$, 该曲线有高的相

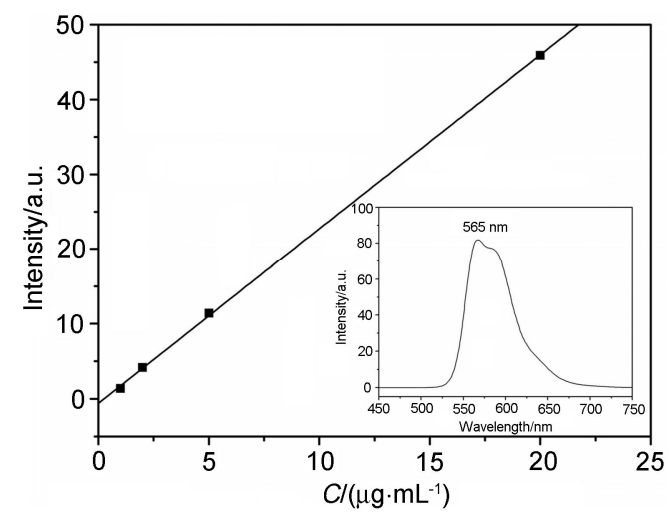

图 $7 \mathrm{DOX}$ 在 $565 \mathrm{~nm}$ 处的标准曲线

Figure 7 Standard concentration curve of DOX at $565 \mathrm{~nm}$ Inset is fluorescence spectrum of DOX 
关系数 $(R=0.9997)$ 和低的标准偏差 $(\mathrm{SD}=0.00473)$, 说 明得到的是一条有效的荧光强度一浓度标准曲线.

将所得到的载药胶束水溶液经冷冻干燥, 得到固态 的载药胶束 6 , 再以一定量的 DMF 溶解, 配制成一定浓 度的载药胶束/DMF 溶液, 测试其在 $565 \mathrm{~nm}$ 处的苂光强 度, 对照标准曲线计算其浓度. 进而计算得到聚合物胶 束的载药量(Drug Loading Content, DLC)和包封率(drug loading efficiency, DLE) 分别为 5.4\%和 $41.25 \%$. 动态光 散射(DLS)结果显示, 载药后的胶束尺寸约 $118 \mathrm{~nm}$, 说 明其能通过 EPR 效应对肿瘤组织进行被动靶向.

\section{2 结论}

以 PEG 为起始物, 通过在其末端连上链转移试剂, 接着利用 RAFT 和 CROP 活性聚合技术, 分别引入 $\mathrm{P} t \mathrm{BA}$ 和 PLA 链段, 得到接枝共聚物 PEG- $b$-(PtBA- $g$-PLA). 随 后, 将苂光标记分子 7-甲氧基香豆素-3-羧酸通过酯化反 应引入到接枝共聚物 PEG- $b$-(PtBA- $g$-PLA), 得到的聚合 物进行选择性水解主链上的叔丁基, 进而制得含荧光标 记分子的两亲性接枝共聚物 PEG- $b$-(PAA- $g$-PLA-COU). 该聚合物可对阿霉素进行有效的负载, 制得载药量较高 的含荧光标记分子的聚合物载药胶束.

\section{3 实验部分}

\section{1 仪器与试剂}

核磁共振: 超导核磁共振仪, 在 Varian MERCURY 300, Bruker AM-300 和 DPX 300 上完成. 溶剂为氞代氯 仿和氛代甲醇, 四甲基硅(TMS)为内标. 红外光谱: 傅 立叶变换红外光谱仪, Nicolet AVATAR-360. 所有红外 谱图累加 32 次, 分辨率为 $4.0 \mathrm{~cm}^{-1}$. 凝胶色谱法测定聚 合物分子量及分子量分布: 实验仪器为 Waters 1515 凝 胶色谱仪(HR3, HR4, HR5), 采用 Waters 2410 示差折光 检测器和 Waters 2487 紫外检测器. 用 THF 将聚合物配 成 $1 \mathrm{mg} / \mathrm{mL}$ 的溶液, 淋洗剂为 THF, 流速为 $1 \mathrm{~mL} / \mathrm{min}$, 采用线性聚苯乙烯标样对分子量进行校正. 苂光光谱: 在 Hitachi F-2700 荧光分光光度计上完成. 动态光散射 粒径仪: Marlven Nano-ZS90 Zetasizer, 溶液过 $0.45 \mu \mathrm{m}$ 滤膜, $25^{\circ} \mathrm{C}$ 下平衡 $2 \mathrm{~min}$, 采集粒径数据.

偶氮二异丁腈(azobisisobutyronitrile, AIBN)用乙醇 $35{ }^{\circ} \mathrm{C}$ 重结晶两次后, $25{ }^{\circ} \mathrm{C}$ 减压干燥 $1 \mathrm{~d}$, 避光保存. $\mathrm{N}, \mathrm{N}$-二甲基甲酰胺(DMF)先经 $\mathrm{NaOH}$ 处理 $10 \mathrm{~h}$, 然后在 无水 $\mathrm{CaSO}_{4}$ 存在下减压蒸出. 4-二甲氨基吡啶(4-Dimethylaminopyridine, DMAP)用干燥的甲苯重结晶三次 后, 真空干燥箱烘干. 外消旋丙交酯 ( $D L$-LA) 用乙酸乙 酯重结晶三次, 真空干燥箱烘干. 氯仿用浓 $\mathrm{H}_{2} \mathrm{SO}_{4}$ 洗三 次, 水洗三次, 再饱和食盐水洗三次后, $\mathrm{CaH}_{2}$ 干燥回流,
现蒸现用. 二氯甲烷经 $\mathrm{CaH}_{2}$ 干燥回流后蒸出, 现蒸现 用，未经纯化直接使用。7-甲氧基香豆素-3-羧酸 (Aldrich，97\%)，二环已基碳二亚胺 (DCC, Aldrich, 99.0\%), 三氟乙酸(TFA, Aldrich, 99.0\%)和 4-氧基-4-(十 二烷硫基硫代羰基)硫基戊酸(Aldrich)和阿霉素(DOX, 浙江海正药业有限公司, 99\%)均未经处理直接使用. $t$ BHBMA $^{[56]}$ 和 $m-\mathrm{PEG}_{2000}-\mathrm{CTA} \mathbf{1}^{[51,52]}$ 按照文献中的方法 合成.

\section{2 实验方法}

\subsection{1 大分子引发剂 PEG- $b$-P $t$ BHBMA (2)的合成}

将加有磁子的橡胶塞密封的 $10 \mathrm{~mL}$ Schlenk 管火烤、 抽换氮气三次; 氮气氛围下加入 AIBN $(8 \mathrm{mg}, 0.049$ $\mathrm{mmol}$ )和 $m-\mathrm{PEG}_{2000} \mathrm{CTA}$ (1) (351 mg, $\left.0.15 \mathrm{mmol}\right)$, 抽换 氮气三次; 充氮气条件下注射器加入 $t$ BHBMA 单体 $(0.6 \mathrm{~mL}, 3.9 \mathrm{mmol})$ 和新蒸的 DMF $(0.6 \mathrm{~mL})$. 液氮冻住体 系后, 油原抽 $10 \mathrm{~min}$, 充氮气解冻再液氮冻住, 重复操 作三次. 将体系投入预设的 $70{ }^{\circ} \mathrm{C}$ 油浴中反应 $30 \mathrm{~h}$. 体 系浸入液氮冻住后, 打开橡皮塞, 通大气后解冻. THF 稀释体系后正己烷冰盐浴中沉淀, 重复操作三次, 真空 干燥后得到 $0.5 \mathrm{~g}$ 黄色透明粘稠液体 PEG- $b$-P $t$ BHBMA (2). GPC: $M_{\mathrm{n}}=6200 \mathrm{~g} / \mathrm{mol}, M_{\mathrm{w}} / M_{\mathrm{n}}=1.19 .{ }^{1} \mathrm{H}$ NMR (400 $\left.\mathrm{MHz}, \mathrm{CD}_{3} \mathrm{OD}\right) \delta: 0.87\left(3 \mathrm{H}, \mathrm{CH}_{2} \mathrm{CH}_{3}\right), 1.29\left[18 \mathrm{H},\left(\mathrm{CH}_{2}\right)_{9}\right]$, $1.51\left(3 \mathrm{H}, \mathrm{CCH}_{3}\right), 1.84\left(3 \mathrm{H}, \mathrm{CNCCH}_{3}, \mathrm{HOCH}_{2} \mathrm{CH}_{2}\right)$, $2.16 \sim 2.48\left(2 \mathrm{H}, \mathrm{CH}_{2} \mathrm{COO} ; 2 \mathrm{H}, \mathrm{CH}_{2} \mathrm{CCN}\right), 3.35(2 \mathrm{H}$, $\left.\mathrm{SCH}_{2} \mathrm{CH}_{2}\right), 3.54 \sim 3.86\left(4 \mathrm{H}, \mathrm{OCH}_{2} \mathrm{CH}_{2} \mathrm{O} ; 3 \mathrm{H}, \mathrm{OCH}_{3} ; 2 \mathrm{H}\right.$, $\left.\mathrm{CH}_{2} \mathrm{OH}\right), 4.23\left(2 \mathrm{H}, \mathrm{COOCH}_{2}\right)$.

\subsection{2 接枝共聚物 PEG- $b$-(PtBA- $g$-PLA) (3) 的合成}

加有磁子的橡胶塞密封的 $10 \mathrm{~mL}$ Schlenk 管火烤、 抽换氮气四次; 氮气氛围下快速加入 PEG- $b$-P $t$ BHBMA (2) $(110 \mathrm{mg}, 0.23 \mathrm{mmoL}-\mathrm{OH}) 、$ DMAP $(0.56 \mathrm{~g}, 0.46$ $\mathrm{mmol})$ 和 DL-LA $(0.5 \mathrm{~g}, 3.47 \mathrm{mmol})$, 抽换氮气五次; 用 抽换好氮气的干燥注射器快速加入新蒸的 $\mathrm{CHCl}_{3}(2.5$ $\mathrm{mL}$ ), 投入预设的 $60{ }^{\circ} \mathrm{C}$ 油浴反应 $24 \mathrm{~h}$. 抽除部分溶剂后, 甲醇冰盐浴中沉淀, 过滤后用 $\mathrm{CHCl}_{3}$ 溶解, 再次沉淀, 重复多次, 真空干燥后得到白色粉末状固体 PEG- $b$-(PtBA-g- PLA) 3240 mg. GPC: $M_{\mathrm{n}}=10,400$ $\mathrm{g} / \mathrm{mol}, M_{\mathrm{w}} / M_{\mathrm{n}}=1.18 .{ }^{1} \mathrm{H} \mathrm{NMR}\left(400 \mathrm{MHz}, \mathrm{CDCl}_{3}\right) \delta: 0.81$ $\left(3 \mathrm{H}, \mathrm{CH}_{2} \mathrm{CH}_{3}\right), 1.23\left[18 \mathrm{H},\left(\mathrm{CH}_{2}\right)_{9}\right], 1.42\left(3 \mathrm{H}, \mathrm{CCH}_{3}\right), 1.55$ $\left[\mathrm{C}\left(\mathrm{CH}_{3}\right)_{3}\right], 1.82,1.93\left(2 \mathrm{H}, \mathrm{CH}_{2} \mathrm{CH}_{2} \mathrm{COO} ; 2 \mathrm{H}, \mathrm{CCH}_{2} \mathrm{C}\right), 2.4$ $\left(2 \mathrm{H}, \mathrm{CH}_{2} \mathrm{COO}\right), 3.35\left(2 \mathrm{H}, \mathrm{SCH}_{2} \mathrm{CH}_{2}\right), 3.51 \sim 3.72(4 \mathrm{H}$, $\left.\mathrm{OCH}_{2} \mathrm{CH}_{2} \mathrm{O} ; 3 \mathrm{H}, \mathrm{OCH}_{3}\right), 4.16 \sim 4.32\left(2 \mathrm{H}, \mathrm{COOCH}_{2} ; 1 \mathrm{H}\right.$, $\left.\mathrm{HOCHCH}_{3}\right), 5.14(1 \mathrm{H}, \mathrm{COOCHCOO})$.

3.2.3 荧光分子标记的两亲性接枝共聚物 PEG- $b$ (PtBA-g-PLA-COU) (4) 的合成

将加有磁子的 $25 \mathrm{~mL}$ 三颈瓶烘烤抽换氮气三次, 加 


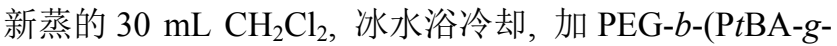
PLA) 3 (1.2 g, $1.5 \mathrm{mmol}$ ), DMAP (18 mg, $0.15 \mathrm{mmol}$ )和 7甲氧基香豆素-3-羧酸 $(50 \mathrm{mg}, 0.5 \mathrm{mmol}$ ), 搅拌下加 DCC (220 mg, $1 \mathrm{mmol})$, 冰浴条件下继续反应 $2 \mathrm{~h}$, 撤除冰浴, 自然恢复室温后继续反应 $24 \mathrm{~h}$. 滤除固体, $\mathrm{CH}_{2} \mathrm{Cl}_{2}$ 洗涤 滤饼, 滤液浓缩后冰乙醚中沉淀, 反复溶解沉淀多次, 真空干燥后得到淡黄色透明粘稠液体 $700 \mathrm{mg}$. GPC: $M_{\mathrm{n}}=10900 \mathrm{~g} / \mathrm{mol}, M_{\mathrm{w}} / M_{\mathrm{n}}=1.17 .{ }^{1} \mathrm{H}$ NMR $(400 \mathrm{MHz}$, $\left.\mathrm{CDCl}_{3}\right) \delta: 0.81\left(3 \mathrm{H}, \mathrm{CH}_{2} \mathrm{CH}_{3}\right), 1.23\left[18 \mathrm{H},\left(\mathrm{CH}_{2}\right)_{9}\right], 1.42$ $\left[\mathrm{C}\left(\mathrm{CH}_{3}\right)_{3}\right], 1.55\left(3 \mathrm{H}, \mathrm{CCH}_{3}\right), 1.82,1.93\left(2 \mathrm{H}, \mathrm{CH}_{2} \mathrm{CH}_{2} \mathrm{CO}-\right.$ $\left.\mathrm{O} ; 2 \mathrm{H}, \mathrm{CCH}_{2} \mathrm{C}\right), 2.4\left(2 \mathrm{H}, \mathrm{CH}_{2} \mathrm{COO}\right), 3.35\left(2 \mathrm{H}, \mathrm{SCH}_{2} \mathrm{CH}_{2}\right)$, $3.51 \sim 3.72\left(4 \mathrm{H}, \mathrm{OCH}_{2} \mathrm{CH}_{2} \mathrm{O} ; 3 \mathrm{H}, \mathrm{OCH}_{3}\right), 3.91[3 \mathrm{H}$, $\left.(\mathrm{CH})_{2} \mathrm{COCH}_{3}\right], 4.16 \sim 4.32\left(2 \mathrm{H}, \mathrm{COOCH}{ }_{2} ; 1 \mathrm{H}, \mathrm{HOCH}-\right.$ $\left.\mathrm{CH}_{3}\right), 5.14(1 \mathrm{H}, \mathrm{COOCHCOO}), 5.35\left[1 \mathrm{H}, \mathrm{OCO}\left(\mathrm{CH}_{3}\right) \mathrm{CH}-\right.$ $\left.\mathrm{OCOC}_{9} \mathrm{H}_{4} \mathrm{O}_{2}\right], 6.82,6.92,7.53,8.59\left(4 \mathrm{H}, \mathrm{C}_{9} \mathrm{H}_{4} \mathrm{O}_{2}\right)$.

\subsubsection{PEG- $b-(\mathrm{P} t \mathrm{BA}-g-\mathrm{PLA}-\mathrm{COU})$ (4) 的选择性水解}

将 PEG- $b$-(PtBA- $g$-PLA-COU) (4) (700 mg, $M_{\mathrm{n}}=$ $\left.10900 \mathrm{~g} / \mathrm{mol}, M_{\mathrm{w}} / M_{\mathrm{n}}=1.17\right)$ 和干燥的 $\mathrm{CH}_{2} \mathrm{Cl}_{2}(120 \mathrm{~mL})$ 加入 $250 \mathrm{~mL}$ 三颈瓶, 冰水浴冷却后加入 TFA $(0.2 \mathrm{~mL})$. $0{ }^{\circ} \mathrm{C}$ 条件下反应 $2 \mathrm{~h}$, 室温下继续反应 $24 \mathrm{~h}$. 体系浓缩后 甲醇冰盐浴中沉淀过滤, 重复多次后, 真空干燥得到淡 黄色固体 PEG- $b$-(PAA- $g$-PLA)-COU (5) $660 \mathrm{mg}$. $M_{\mathrm{n}}=$ $10600 \mathrm{~g} / \mathrm{mol}, M_{\mathrm{w}} / M_{\mathrm{n}}=1.18 .{ }^{1} \mathrm{H}$ NMR $\left(400 \mathrm{MHz}, \mathrm{CDCl}_{3}\right)$ $\delta: 0.81\left(3 \mathrm{H}, \mathrm{CH}_{2} \mathrm{CH}_{3}\right), 1.23\left[18 \mathrm{H},\left(\mathrm{CH}_{2}\right)_{9}\right], 1.55(3 \mathrm{H}$, $\left.\mathrm{CCH}_{3}\right), 1.82,1.93\left(2 \mathrm{H}, \mathrm{CH}_{2} \mathrm{CH}_{2} \mathrm{COO} ; 2 \mathrm{H}, \mathrm{CCH}_{2} \mathrm{C}\right), 2.4$ $\left(2 \mathrm{H}, \mathrm{CH}_{2} \mathrm{COO}\right), 3.35\left(2 \mathrm{H}, \mathrm{SCH}_{2} \mathrm{CH}_{2}\right), 3.51 \sim 3.72(4 \mathrm{H}$, $\left.\mathrm{OCH}_{2} \mathrm{CH}_{2} \mathrm{O} ; 3 \mathrm{H}, \mathrm{OCH}_{3}\right), 3.91\left[3 \mathrm{H},(\mathrm{CH})_{2} \mathrm{COCH}_{3}\right], 4.16 \sim$ $4.32\left(2 \mathrm{H}, \mathrm{COOCH}_{2} ; 1 \mathrm{H}, \mathrm{HOCHCH}_{3}\right), 5.14(1 \mathrm{H}, \mathrm{COOCH}-$ $\mathrm{COO}), 5.35\left[1 \mathrm{H}, \mathrm{OCO}\left(\mathrm{CH}_{3}\right) \mathrm{CHOCOC}_{9} \mathrm{H}_{4} \mathrm{O}_{2}\right], 6.82,6.92$, $7.53,8.59\left(4 \mathrm{H}, \mathrm{C}_{9} \mathrm{H}_{4} \mathrm{O}_{2}\right)$.

\subsubsection{PEG- $b$-(PAA- $g$-PLA)-COU (5) 对 DOX 的负载}

首先称取 $30 \mathrm{mg}$ 阿霉素盐酸盐溶于 $3 \mathrm{~mL} \mathrm{DMF}$ 中, 搅拌下加入 $30 \mu \mathrm{L} \mathrm{Et}_{3} \mathrm{~N}$, 避光搅拌过夜. 再称取 $10 \mathrm{mg}$ PEG- $b$-(PAA- $g$-PLA)-COU (5)溶于 $1 \mathrm{~mL}$ THF, 充分溶解 后向其中加入 $100 \mu \mathrm{L}$ 新配制的 $10 \mathrm{mg} / \mathrm{mL}$ DOX/DMF 溶 液, 继续摚拌 $2 \mathrm{~h}$. 将所得混合液在摚拌下滴加入 $10 \mathrm{~mL}$ 双蒸水中, 继续室温搅拌 $12 \mathrm{~h}$ 使部分 THF 挥发, 转移至 透析袋 $($ cutoff $=3000)$ 中, $400 \mathrm{~mL}$ 双蒸水中透析 $24 \mathrm{~h}$, 每 $3 \mathrm{~h}$ 更换一次双蒸水. 最后负载 DOX 的胶束溶液冷冻 干燥, 得负载 DOX 的含荧光标记分子的聚合物载药胶 束 6. 称取定量的聚合物载药胶束 6 用 DMF 将其溶解, 定容至 $50 \mathrm{~mL}$. 运用苂光光谱测定 DOX 在 $565 \mathrm{~nm}$ 处的 苂光强度(激发波长 $538 \mathrm{~nm}$ ). 进一步通过 DOX 浓度-苂 光强度工作曲线计算出 DOX 的含量, 从而计算得到聚 合物胶束对 DOX 的载药量和包封率.
辅助材料(Supporting Information) 聚合物 PEG- $b$ P $t$ BHBMA (2)、PEG- $b-(\mathrm{P} t \mathrm{BA}-g-\mathrm{PLA})(3) 、 \mathrm{PEG}-b$-(PtBA$g$-PLA-COU) (4) 和 PEG- $b$-(PAA- $g$-PLA)-COU (5)的核磁 氢谱; PEG- $b$-(PAA- $g$-PLA-COU) (5) 的 DMF 溶液的荧光 光谱; DOX 的荧光光谱等原始数据. 这些材料可以免费 从本刊网站(http://sioc-journal.cn/)上下载.

\section{References}

[1] Fylaktakidou, K. C.; Hadjipavlou-Litina, D. J.; Litinas, K. E.; Nicolaides, D. N. Curr. Pharm. Des. 2004, 10, 3813.

[2] Walshe, M.; Howarth, J.; Kelly, M. T.; O'Kennedyc, R,; Smyth, M. R. J. Pharm. Biomed. Anal. 1997, 16, 319.

[3] (a) Sardari, S.; Mori, Y.; Horita, K.; Micetich, R. G.; Nishibe, S.; Daneshtalab, M. Bioorg. Med. Chem. 1999, 7, 1933.

(b) Chen, H.; Zhou, L. K.; Li, S.; Yao, Y. C.; Gu, Y. J.; Li, C. X.; Li, N.; Meng, M.; Li, X. H. Chin. J. Org. Chem. 2013, 33, 164 (in Chinese).

(陈华, 周利凯, 李帅, 姚玉超, 谷云景, 李春晓, 李娜, 孟明, 李 小六, 有机化学, 2013, 33, 164.)

[4] Trenor, S. R.; Shultz, A. R.; Love, B. J.; Long, T. E. Chem. Rev. 2004, 104, 3059.

[5] Weber, U. S.; Steffen, B.; Siegers, C. P. Res. Commun. Mol. Pathol. Pharmacol. 1998, 99, 193.

[6] Grifths, J.; Millar, V. Dyes Pigm. 1995, 28, 327.

[7] Cao, X.; Lin, W.; Yu, Q.; Wang J. Org. Lett. 2011, 13, 6098.

[8] Kim, I.; Kim, D.; Sambasivan, S.; Ahn, K. H. Asian J. Org. Chem. 2012, 1,60 .

[9] Cao, X.; Lin, W.; Yu, Q. J. Org. Chem. 2011, 76, 7423.

[10] Ray, D.; Bharadwaj, P. K. Inorg. Chem. 2008, 47, 2252.

[11] (a) Zhu, W. H.; Tian, H.; Zhu, S. Q. Prog. Chem. 2002, 14, 18 (in Chinese).

(朱为宏, 田禾, 朱世琴, 化学进展, 2002, 14, 18.)

(b) Han, L.; Zhou, X.; Ye, Q.; Li, Y. J.; Gao, J. R. Chin. J. Org. Chem. 2013, 33, 1000 (in Chinese).

(韩亮, 周雪, 叶青, 李郁锦, 高建荣, 有机化学, 2013, 33, 1000.)

[12] Hung, T. T.; Lu, Y. J.; Liao, W. Y.; Huang, C. L. IEEE T. Magn. 2007, 43, 867.

[13] Hara, K.; Sayama, K.; Ohga,Y.; Shinpo, A.; Suga, S.; Arakawa, H. Chem. Commun. 2001, 6, 569.

[14] Fan, J. L.; Xu, Q. L.; Zhu, H.; Peng, X. J. Chin. J. Org. Chem. 2014, 34, 1623 (in Chinese). (訤江莉, 徐群利, 朱浩, 彭孝军, 有机化学, 2014, 34, 1623.)

[15] (a) Tang, C. B.; Lennon, E. M.; Fredrickson, G. H.; Kramer, E. J.; Hawker, C. J. Science 2008, 322, 429.

(b) Cai, X. B.; Hu, W.; Lu, G. L.; Huang, X. Y. Chin. J. Org. Chem. 2013, 33, 2520 (in Chinese).

(蔡晓冰，胡薇，陆国林，黄晓宇，有机化学, 2013, 33, 2520.)

[16] Taton, K. S.; Guire, P. E. Biointerfaces 2002, 24, 123.

[17] Liu, T.; Zhang, Y. F.; Liu, S. Y. Chin. J. Polym. Sci. 2013, 31, 924.

[18] Kataoka, K.; Harada, A.; Nagasaki, Y. Adv. Drug Delivery Rev. 2001, 47, 113.

[19] Moeser, G. D.; Green, W. H.; Laibinis, P. E.; Linse, P.; Hatton, T. A. Langmuir 2004, 20, 5223.

[20] Zhang, M. F.; Estourne, C.; Bietsch, W.; Muller, A. H. E. $A d v$. Funct. Mater. 2004, 14, 871.

[21] Lutz, J. F.; Stiller, S.; Hoth, A.; Kaufner, L.; Pison, U.; Cartier, R. Biomacromolecules 2006, 7, 3132.

[22] Gu, L. N.; Shen, Z.; Feng, C.; Li, Y. G.; Lu, G. L.; Huang, X. Y.; Wang, G. W.; Huang, J. L. J. Mater. Chem. 2008, 18, 4332. 
[23] Brannon-Peppas, L.; Blanchette, J. O. Adv. Drug Delivery Rev. 2004, 56, 1649

[24] Smyth, H. F.; Carpenter, C. P.; Weil, C. S. J. Am. Pharm. Assoc. Sci. Ed. 1955, 44, 27.

[25] Leung, H. W.; Ballantyne, B.; Hermansky, S. J.; Franta, S. W. Int. J. Toxicol. 2000, 19, 305.

[26] Pang, S. N. J. J. Am. Coll. Toxicol. 1993, 12, 429.

[27] Torchilin, V. P. Adv. Drug Delivery Rev. 2002, 54, 235.

[28] Hu, Y.; Jiang, X.; Ding, Y. Biomaterials 2003, 24, 2395.

[29] Wood, D. Int. J. Pharm. 1980, 7, 1.

[30] Kulkarni, R. K., Pani, K. C. Arch. Surg. 1966, 93, 839.

[31] Mori, H.; Müller, A. H. E. Prog. Polym. Sci. 2003, 28, 1403.

[32] Guyot, A.; Tauer, K. Adv. Polym. Sci. 1994, 111, 43.

[33] Kikuchi, A.; Nose, T. Macromolecules 1997, 30, 896.

[34] Teertstra, S. J.; Gauthier, M. Prog. Polym. Sci. 2004, 29, 277.

[35] Yamada, K.; Miyazaki, M.; Ohno, K.; Fukuda, T.; Minoda, M. Macromolecules 1999, 32, 101.

[36] Lligadas, G.; Rosen, B. M.; Bell, C. A.; Monteiro, M. J.; Percec, V. Macromolecules 2008, 41, 8365 .

[37] Nguyen, N. H.; Rosen, B. M.; Lligadas, G.; Percec, V. Macromolecules 2009, 42, 2379.

[38] Deffieux, A.; Schappacher, M. Macromolecules 1999, 32, 1797.

[39] Park, S. Y.; Han, B, R.; Na, K. M.; Han, D. K.; Kim, S. C. Macromolecules 2003, 36, 4115.

[40] Moses, J. E.; Moorhouse, A. D. Chem. Soc. Rev. 2007, 36, 1249.

[41] Li, G.; Wang, H.; Zheng, H. T.; Bai, R. K. J. Polym. Sci., Polym. Chem. 2010, 48, 1348.

[42] Xu, X. W.; Huang, J. L. J. Polym. Sci., Polym. Chem. 2004, 42,
5523.

[43] Grubbs, R. B.; Hawker, C. J.; Dao, J.; Frechet, J. M. J. Angew. Chem., Int. Ed. 1997, 36, 270.

[44] Wang, J. S.; Matyjaszewski, K. J. Am. Chem. Soc. 1995, 117, 5614.

[45] Siegwart, D. J.; Oh, J. K.; Matyjaszewski, K. Prog. Polym. Sci. 2012, 37, 18 .

[46] Percec, V.; Guliashvili, T.; Ladislaw, J. S.; Wistrand, A.; Stjerndahl, A.; Sienkowska, M. J.; Monteiro, M. J.; Sahoo, S. J. Am. Chem. Soc. 2006, 128, 14156.

[47] Rosen, B. M.; Percec, V. Chem. Rev. 2009, 109, 5069.

[48] Cheng, Z. P.; Zhu, X. L.; Fu, G. D.; Kang, E. T.; Neoh, K. G. Macromolecules 2005, 38, 7187.

[49] Liu, J. N.; Duong, H.; Whittaker, M. R.; Davis, T. P.; Boyer, C. Macromol. Rapid Commun. 2012, 33, 760.

[50] Qian, W. H.; Xu, P. C.; Lu, G. L.; Huang, X. Y. Chin. J. Chem. 2014, 32, 1049.

[51] Chong, Y. K.; Le, T. P. T.; Moad, G.; Rizzardo, E.; Thang, S. H. Macromolecules 1999, 32, 2071.

[52] Achilleos, M.; Legge, T. M.; Perrier, S.; Patrickios, C. S. J. Polym. Sci., Part A: Polym. Chem. 2008, 46, 7556.

[53] Allen, T. M.; Cullis, P. R. Science 2004, 303, 1818.

[54] Chen, H.; Yuan, L.; Song, W.; Wu, Z.; Li, D. Prog. Polym. Sci. 2008, 33, 1059.

[55] Hashida, M.; Nishikawa, M.; Takakura, Y. J. Controlled Release 1995, 36, 99.

[56] Song, X. M.; Yao, W. Q.; Lu, G. L.; Li,Y. J.; Huang, X. Y. Polym. Chem. 2013, 4, 2864.

(Cheng, F.) 